Pacific Journal of Mathematics

SOME CHARACTERIZATIONS OF ANALYTIC METRIC
SPACES 


\title{
SOME CHARACTERIZATIONS OF ANALYTIC METRIC SPACES
}

\author{
SOPHOCLES MERCOURAKIS
}

\begin{abstract}
For a topological space $X$, let $C_{1}(X)$ denote the Banach space of all bounded functions $f: X \rightarrow \mathbf{R}$ such that for every $\varepsilon>0$ the set $\{x \in X$ : $|f(x)| \geq \varepsilon\}$ is closed and discrete in $X$, endowed with the supremum norm. Using spaces of this form we give a direct proof (Corollary 1.5) of a result of Dashiell and Lindenstrauss on strict convexity of Banach spaces. Subsequently we obtain two types of characterizations of analytic metric spaces. The first (Theorem 2.3) is topological and is based on the set theoretic ordering of the compact subsets of $X$; this is related to some results of Christensen and Talagrand. The second (Theorem 3.1) is functional analytic and is based on the existence of bounded linear operators between the spaces of the form $C_{1}(X)$.
\end{abstract}

1. A simple example of Dashiell-Lindenstrauss type. In this section we introduce the class of Banach spaces $C_{1}(X)$, where $X$ is a topological space and give a simple proof that there is no bounded linear one-to-one operator from $C_{1}(\Sigma)$, where $\Sigma$ is the Baire space $\omega^{\omega}$ (of infinite sequences of natural numbers with the product topology) into the classical Banach space $C_{0}(\Gamma)=\{f: \Gamma \rightarrow \mathbf{R}$ : for every $\varepsilon>0$ the set $\{\gamma \in \Gamma:|f(\gamma)| \geq \varepsilon\}$ is finite $\}$, for any set $\Gamma$. Since $C_{1}(\Sigma)$ is strictly convexifiable $[7,8]$ the Banach space $C_{1}(\Sigma)$ is still another example with the properties of the examples of Dashiell-Lindenstrauss (see [2], Theorem 2).

Definition 1.1. Let $X$ be a topological space. We set $C_{1}(X)=\{f$ : $X \rightarrow \mathbf{R}: f$ is bounded and for every $\varepsilon>0$ the set $\{t \in X:|f(t)| \geq \varepsilon\}$ is closed and discrete in $X\}$. It is clear that $C_{1}(X)$ with supremum norm is a Banach space.

We notice that:

(a) For a subset $A \subseteq X, A$ is closed and discrete in $X$ if and only if $A^{\prime}$, the derived set of $A$, is empty;

(b) $C_{0}(X) \subseteq C_{1}(X)$, and if $f \in C_{1}(X)$ then $f \mid \Omega \in C_{0}(X)$ for all compact subsets $\Omega$ of $X$;

(c) if $A$ is compact (resp. closed and discrete) in $X$, then $C_{0}(A)$ (resp. $l^{\infty}(A)$ is a complemented subspace of $\left.C_{1}(X)\right)$. In particular, if $X$ is compact (resp. discrete) then $C_{1}(X)=C_{0}(X)\left(\right.$ resp. $\left.C_{1}(X)=l^{\infty}(X)\right)$; 
(d) if $Y$ is a closed subset of $X$ then $C_{1}(Y)$ is naturally embedded in $C_{1}(X)$; indeed, since $Y$ is closed in $X, A \subseteq Y$ is closed and discrete in $Y$ if and only if $A$ is closed and discrete in $X$.

REMARK 1.2. The class of Banach spaces of the form $C_{1}(X)$ were introduced in [7], for the study of weakly countably determined (WCD) Banach spaces. In $[7,8]$ is proved that, if $\Omega$ is compact space then the Banach space $C(\Omega)$ is WCD if and only if there exist a subset $\Sigma^{\prime} \subseteq \Sigma$ a compact space $K$ and a bounded linear one-to-one operator $T$ from the space on Radon measures $M(\Omega)$ on $\Omega$ into $C_{1}\left(\Sigma^{\prime} \times K\right)$ that is weak* to pointwise continuous. Similar results hold for weakly $K$-analytic Banach spaces.

In this paper the space $X$ will be a separable metric space. Thus, $C_{1}(X)$ related to the classical Banach space $C_{0}(X)$, in the same way in which a compact metric space is related to a separable one.

The next lemma is the key lemma of $\S \S 1$ and 3. For the proof we need some preliminaries. A Banach space $E$ is Grothendieck, if the weak and weak* convergence of sequences in $E^{*}$ are the same. It is well known that the classical Banach space $l^{\infty}(A)$, for any set $A$ is Grothendieck (see [5], p. 168). Notice also that this property of $l^{\infty}(A)$, implies that every bounded linear operator $F$ from $l^{\infty}(A)$ into $C_{0}(\Gamma)$, for any set $\Gamma$, is weakly compact, that is $F\left(\left\{x \in l^{\infty}(A)\right.\right.$ : $\left.\left.\|x\| \leq 1\right\}\right)$ is weakly relatively compact in $C_{0}(\Gamma)$ (see [3], pp. 143 and 150).

LEMMA 1.3. Let $X$ be a separable metric space, $\Gamma$ be a set and $T$ : $C_{1}(X) \rightarrow C_{0}(\Gamma)$ a bounded linear operator. Then for every $\varepsilon>0$, the set $\left\{t \in X:\left\|T\left(\chi_{\{t\}}\right)\right\| \geq \varepsilon\right\}$ is relatively compact in $X$.

Proof. Set for every $\varepsilon>0, X_{\varepsilon}=\left\{t \in X:\left\|T\left(\chi_{\{t\}}\right)\right\| \geq \varepsilon\right\}$. Suppose (for the purpose of contradiction) that some $X_{\varepsilon}$ is not relatively compact. Then there is an infinite subset $A$ of $X_{\varepsilon}$, which is closed and discrete in $X$. We identify $l^{\infty}(A)$ with the subspace $\left\{f \in C_{1}(X): \operatorname{supp}(f) \subseteq A\right\}$ of $C_{1}(X)$ and consider the restriction $F: l^{\infty}(A) \rightarrow C_{0}(\Gamma)$ of $T$ on $l^{\infty}(A)$. By the above remarks $F$ is a weakly compact operator. If we set $F_{1}=$ $F \mid C_{0}(A): C_{0}(A) \rightarrow C_{0}(\Gamma)$, then $F$ is weakly compact, so the dual operator $F_{1}{ }^{*}: l^{1}(\Gamma) \rightarrow l^{1}(A)$ is weakly compact as well (see [4]). Since $l^{1}(A)$ has the Schur property $\left([\mathbf{1 1}]\right.$, p. 122), $F_{1}{ }^{*}$ is a compact operator and, by [4], $F_{1}$ is compact as well.

Let $\left\{t_{m}: m<\omega\right\}$ be an enumeration of $A$. Because $\chi_{\left\{t_{m}\right\}} \rightarrow 0$ weakly in $C_{0}(A), F\left(\chi_{\left\{t_{m}\right\}}\right)=F_{1}\left(\chi_{\left\{t_{m}\right\}}\right) \rightarrow 0$ weakly in $C_{0}(\Gamma)$. Because $F_{1}$ is 
compact, $\left\|F\left(\chi_{\left\{t_{m}\right\}}\right)\right\| \rightarrow 0$. But this is impossible, because $\left\|F\left(\chi_{\left\{t_{m}\right\}}\right)\right\|=$ $\left\|T\left(\chi_{\left\{t_{m}\right\}}\right)\right\| \geq \varepsilon$, for all $m<\omega$.

THEOREM 1.4. For a metric space $X$ the following are equivalent:

(a) $X$ is $\sigma$-compact;

(b) there exist a set $\Gamma$ and a bounded linear one-to-one operator $T$ : $C_{1}(X) \rightarrow C_{0}(\Gamma)$, which is continuous when $C_{1}(X)$ and $C_{0}(\Gamma)$ are endowed with the topology of pointwise convergence;

(c) there exist a set $\Gamma$ and a bounded linear operator $T: C_{1}(X) \rightarrow C_{0}(\Gamma)$ such that $T\left(\chi_{\{t\}}\right) \neq 0$ for all $t \in X$.

Proof. (a) $\Rightarrow$ (b) Let $X=\cup_{n<\omega} K_{n}$, where each $K_{n}$ is compact. We set $\Gamma=\cup_{n<\omega}\left(K_{n} \times\{n\}\right)$ and define $T: C_{1}(X) \rightarrow C_{0}(\Gamma)$ by $T(f)(t, n)=$ $f(t) / n$. To see that $T(f) \in C_{0}(\Gamma)$, let $\varepsilon>0$ and set $A=\{n<\omega$ : $|T(f)(t, n)| \geq \varepsilon$ for some $\left.t \in K_{n}\right\}$. For every $(t, n) \in \Gamma$ we have $|T(f)(t, n)|=|f(t)|_{n} \leq\left.\|f\|\right|_{n}$, so $A$ is finite and $\bigcup_{n \in A} K_{n}$ is compact. Therefore $\{(t, n) \in \Gamma:|T(f)(t, n)| \geq \varepsilon\}$ is finite. It is easily seen that $T$ has the desired properties.

(b) $\Rightarrow$ (c) is trivial.

(c) $\Rightarrow$ (a) For every $n<\omega$, we set

$$
X_{n}=\left\{t \in X:\left\|T\left(\chi_{\{t\}}\right)\right\| \geq 1 / n\right\} .
$$

Since $T\left(\chi_{\{t\}}\right) \neq 0$ for every $t \in X$, we have $X=\bigcup_{n<\omega} X_{n}$. By Lemma 1.3 we have that every $X_{n}$ is relatively compact in $X$, so $X$ is $\sigma$-compact.

COROLlaRY 1.5. For any set $\Gamma$, there is no bounded linear one-to-one operator $T: C_{1}(\Sigma) \rightarrow C_{0}(\Gamma)$.

ReMark 1.6. The above Corollary 1.5 should be compared with some examples of Dashiell and Lindenstrauss [2] concerning strictly convexifiable Banach spaces. We recall that a Banach space $E$ is said to be strictly convexifiable if there is an equivalent norm $\|\cdot\|$ on $E$ which is strictly convex (that is, for every $x, y \in E$ with $x \neq y$, such that $\|x\|=\|y\|=1$, we have $\|(x+y) / 2\|<1)$. By a classical result of Day [3] $C_{0}(\Gamma)$ is strictly convexifiable. As noted by V. Klee [3] every Banach space that admits a one-to-one bounded linear operator into some strictly convexifiable space has the same property.

Dashiell and Lindenstrauss proved, by constructing suitable examples (see Theorem 2 of [2]), that the existence of a one-to-one bounded linear operator from $E$ into some $C_{0}(\Gamma)$ is not a necessary condition for $E$ to be strictly convexifiable. 
In $[7,8]$ is proved that $C_{1}(\Sigma)$ (in fact any space $C_{1}(X)$ where $X$ is countably determined topological space) admits an equivalent strictly convex norm (which is moreover pointwise lower-semicontinuous). By Corollary 1.5 it follows that $C_{1}(\Sigma)$ is one more example with the properties of the examples of Dashiell-Lindenstrauss. For a different proof of Corollary 1.5 we refer to $[7,8]$.

2. Topological characterizations. We begin with a simple lemma.

Lemma 2.1. For a function $f: X \rightarrow Y$ between metric spaces, the following are equivalent:

(a) for every closed and discrete subset $A$ of $Y, f^{-1}(A)$ is closed and discrete in $X$;

(b) (i) for every $y \in Y, f^{-1}(\{y\})$ is closed and discrete in $X$, and

(ii) for every (relatively) compact subset $K$ of $X, f(K)$ is relatively compact in $Y$.

Proof. (a) $\Rightarrow$ (b) Since (i) is trivial, we only prove (ii). Let $K$ be a compact of $X$. If $f(K)$ were not relatively compact in $Y, K$ contained an infinite closed and discrete subset, which is absurd.

(b) $\Rightarrow$ (a) Let $A$ be a subset of $Y$ such that $f^{-1}(A)$ is not closed and discrete. Then there is a non-trivial convergent sequence $\left(x_{n}\right)_{n \in \omega}$ in $f^{-1}(A)$. The set $\left\{f\left(x_{n}\right): n \in \omega\right\}$ is infinite (by (i)) relatively compact (by (ii)) and is included in $A$. Therefore $A$ is not closed and discrete.

The above lemma in conjunction with a theorem of Christensen [1], yields the following:

Proposition 2.2. Let $X$ be a Polish space and $Y$ a metric space. Suppose that there is a function $f: X \rightarrow Y 1-1$ and onto such that for every $A \subseteq X, f(A)$ is closed and discrete in $Y$ if and only if $A$ is closed and discrete in $X$. Then $Y$ is a Polish space.

Proof. Let $\mathscr{K}(X)$ and $\mathscr{K}(Y)$ be at the families of non-empty compact subsets of $X$ and $Y$, respectively. Define $F: \mathscr{K}(X) \rightarrow \mathscr{K}(Y)$ with $F(A)=\mathrm{cl}_{y} f(A)$. By Lemma $2.1 F$ is well defined and satisfies the following conditions: (a) if $A, B \in \mathscr{K}(X)$ and $A \subseteq B$, then $F(A) \subseteq F(B)$ and (b) for every compact subset $K$ of $Y$, there exists a compact subset $A$ of $X$ such that $F(A) \supseteq K$ (e.g. $A=\mathrm{cl}_{x}\left(f^{-1}(K)\right)$ ). Now the result follows from [1]. 
In the next theorem we use the following notation. If $\sigma, \tau$ are in $\Sigma$, then $\sigma \leq \tau$ means $\sigma(n) \leq \tau(n)$ for all $n \in \omega$. We also set $\Sigma(\sigma)=\{\tau \in \Sigma$ : $\tau \leq \sigma\}$ for every $\sigma \in \Sigma$ and $S=\{\sigma \mid n: \sigma \in \Sigma, n \in \omega\}$, where $\sigma \mid n=$ $(\sigma(1), \sigma(2), \ldots, \sigma(n))$.

Recall that a metric space $X$ is analytic if $X$ is a continuous image of $\Sigma$.

THEOREM 2.3. For a metric space $X$ the following are equivalent:

(a) $X$ is analytic;

(b) there exists a family $\left\{X_{\sigma}: \sigma \in \Sigma\right\}$ of compact subsets of $X$ such that: (i) $X=\bigcup_{\sigma \in \Sigma} X_{\sigma}$ and (ii) if $\sigma, \rho \in \Sigma$ and $\sigma \leq \rho$, then $X_{\sigma} \subseteq X_{\rho}$ (cf. Prop. 6.13 of [9]);

(c) there is an analytic metric space $Y$ and function from $Y$ onto $X$ such that if $A$ is closed and discrete in $X$, then $f^{-1}(A)$ closed and discrete in $Y$.

Moreover, in (c) $f$ can be chosen to be one-to-one and $Y$ to be $\Sigma$ (if $X$ is uncountable) or a closed discrete subset of $\Sigma$ (if $X$ is countable).

Proof. (a) $\Rightarrow$ (b) Let $f: \Sigma \rightarrow X$ be a continuous surjection. Then the family of the sets $X_{\sigma}=f(\Sigma(\sigma)), \sigma \in \Sigma$, has the desired properties.

(b) $\Rightarrow$ (a) First we prove that $X$ is separable. To do this, we show that every closed and discrete subset $D$ of $X$ is countable. For every $x \in D$, we choose $\sigma_{x} \in \Sigma$ such that $x \in X_{\sigma_{x}}$. Then $D \subseteq \bigcup_{x \in D} X_{\sigma_{x}}$ and it suffices to show that $\left\{\sigma_{x}: x \in D\right\}$ is countable. Suppose that this is not the case and choose a non-trivial convergent sequence $\left\{\sigma_{x_{n}}\right\}$. Then $\bigcup_{n=1}^{\infty} \Sigma\left(\sigma_{x_{n}}\right) \subseteq$ $\Sigma(\rho)$ for some $\rho \in \Sigma$. It follows that $\bigcup_{n=1}^{\infty} X_{\sigma_{x_{n}}} \subseteq X_{\rho}$ and so $\left\{x_{n}: n<\omega\right\}$ $\subseteq X_{\rho}$, which is impossible because $x_{n} \in D, n \in \omega$, and $X_{\rho}$ is compact.

Let $K$ be a metrizable compactification of $X$. Set $X_{s}=\operatorname{cl}_{k}\left(\cup\left\{X_{\sigma}\right.\right.$ : $\sigma \in \Sigma, \sigma$ extends $s\}$ ), for every $s \in S$. By [6], p. 482, it suffices to show that $X=\bigcup_{\sigma \in \Sigma} \cap_{p=1}^{\infty} X_{\sigma \mid n}$. Since " $\subseteq$ " is obvious, we prove only the reverse inclusion. Let $x \in \bigcup_{\sigma \in \Sigma} \bigcap_{n=1}^{\infty} X_{\sigma \mid n}$ and let $\left(U_{n}\right)_{n<\omega}$ be a neighborhood base for $x \in K$. Then there exists $\sigma \in \Sigma$ such that $x \in X_{\sigma \mid n}$ for all $n<\omega$. By the definition of the $X_{s}$, for every $n<\omega$ there exist $\sigma_{n} \in \Sigma$ such that $\sigma_{n}|n=\sigma| n$ and $y_{n} \in X_{\sigma_{n}} \cap U_{n}$. Then $\sigma_{n} \rightarrow \sigma$ (in the metric space $\Sigma)$, so there exists $\rho \in \Sigma$ such that $\left\{\sigma_{n}: n<\omega\right\} \subseteq \Sigma(\rho)$, which implies that $\bigcup_{n \in \omega} X_{\sigma_{n}} \subseteq X_{\rho}$ and $\left\{y_{n}: n<\omega\right\} \subseteq X_{\rho}$. Because $y_{n} \in U_{n}$, $y_{n} \rightarrow x$ and so $x \in X_{\rho} \subseteq X$.

(a) $\Rightarrow$ (c) If $X$ is countable, take $Y$ to be any closed and discrete subset of $\Sigma$ with the same cardinal. Then any $f: Y \rightarrow X$ one-to-one and onto satisfies (c). 
Now assume that $X$ is uncountable, so there is a compact uncountable $K \subseteq X$ ([6], p. 479). Let $g: \Sigma \rightarrow X$ be a continuous surjection. Fix a subset $C$ of $\Sigma$ such that $g \mid C$ is 1-1 and $g(C)=X \backslash K$. Define $f: \Sigma \rightarrow X$ such that $f \mid C=g$ and $f \mid \Sigma \backslash C$ is $1-1$ and $f(\Sigma \backslash C)=K$. We shall prove that $f$ satisfies (c). It is clear that $f$ is one-to-one and $f(\Sigma)=X$. Thus, by Lemma 2.1, it suffices to show that for a compact subset $Z$ of $\Sigma, f(Z)$ is relatively compact in $X$.

Let $\left\{z_{n}\right\}$ be a sequence in $Z$ and set $y_{n}=f\left(z_{n}\right)$. Because $Z$ is compact, we can assume that $z_{n} \rightarrow z \in Z$. Let $M=\left\{n<\omega: z_{n} \in \Sigma \backslash C\right\}$. If $M$ is infinite, then by definition of $f$ the set $\left\{f\left(z_{n}\right): n \in M\right\}$ is an infinite subset of $K$ and so it has an accumulation point. Now assume that $M$ is finite. Then $z_{n} \in C$ and $y_{n}=f\left(z_{n}\right)=g\left(z_{n}\right)$ for all $n \in \omega \backslash M$. Since $g$ is continuous, $y_{n} \rightarrow g(z)$.

(c) $\Rightarrow$ (b) Since $(a) \Rightarrow(b)$, there exists a family $\left\{Y_{\sigma}: \sigma \in \Sigma\right\}$ of compact subsets of $Y$ such that $Y=\bigcup_{\sigma \in \Sigma} Y_{\sigma}$ and if $\sigma \leq \rho$, then $Y_{\sigma} \subseteq Y_{\rho}$. By Lemma 2.1, the family $X_{\sigma}=\operatorname{cl}_{X} f\left(Y_{\sigma}\right), \sigma \in \Sigma$, satisfies (b).

I don't know if the assertions of Theorem 2.3 are equivalent to:

(d) there exists an analytic metric space $Y$ such that $X=\bigcup\left\{X_{K}\right.$ : $K \in \mathscr{K}(Y)\}$ and $X_{K_{1}} \subseteq X_{K_{2}}$ if $K_{1}, K_{2} \in \mathscr{K}(Y)$ and $K_{1} \subseteq K_{2}$, and $X_{K}$ compact for all $K \in \mathscr{K}(Y)$.

3. Functional analytic characterizations. In this section we characterize analytic metric space $X$ with the help of linear operators from $C_{1}(X)$ into $C_{1}(\Sigma)$ using functional analytic arguments in conjunction with Theorem 2.3.

THEOREM 3.1. For a metric space $X$ the following are equivalent:

(a) $X$ is analytic;

(b) there exists an isometry $T: C_{1}(X) \rightarrow C_{1}(\Sigma)$ which is continuous when $C_{1}(X)$ and $C_{1}(\Sigma)$ are endowed with the topology of pointwise convergence;

(c) there exists an analytic metric space $Y$ and a bounded linear operator $T: C_{1}(X) \rightarrow C_{1}(Y)$ such that $T\left(\chi_{\{t\}}\right) \neq 0$ for all $t \in X$.

Proof. (a) $\Rightarrow$ (b) By Theorem 2.3, there exist a closed subset $Y$ of and a bijection $f: Y \rightarrow X$ such that for every closed and discrete subset $A$ of $X, f^{-1}(A)$ is closed and discrete in $Y$. We define $T: C_{1}(X) \rightarrow C_{1}(Y) \subseteq$ $C_{1}(\Sigma)$ with $T(\varphi)=\varphi \circ f$. It is easy to see that $T$ has the desired properties.

(b) $\Rightarrow$ (c) is trivial.

(c) $\Rightarrow$ (a) Since (a) $\Rightarrow$ (b), we can assume $Y=\Sigma$. For every $\sigma \in \Sigma$ 
and $n<\omega$, we set

$$
X_{(\sigma, n)}=\left\{t \in X:\left\|T\left(\chi_{\{t\}}\right) \mid \Sigma(\sigma)\right\| \geq 1 / n\right\}
$$

Because

$$
T\left(\chi_{\{t\}}\right) \neq 0, \quad t \in X, \quad X=\bigcup_{(\sigma, n) \in \Sigma \times \omega} X_{(\sigma, n)}
$$

Clearly if $\sigma \leq \tau$ and $n \leq m$ then $X_{(\sigma, n)} \subseteq X_{(\tau, m)}$. Moreover, by Lemma 1.3 each $X_{(\sigma, n)}$ is relatively compact in $X$. Thus, by Theorem 2.3,X is analytic.

In addition to the characterizations of $\boldsymbol{\sigma}$-compact metric spaces of Theorem 1.4, we have:

THEOREM 3.2. For a metric space $X$ the following are equivalent;

(a) $X$ is $\sigma$-compact;

(b) $X$ is analytic and every pointwise compact subset of $C_{1}(X)$ is Eberlein compact (that is, homeomorphic to a weakly compact subset of Banach space).

Proof. (a) $\Rightarrow$ (b). This is immediate, from Theorem 1.4. Since every pointwise compact subset of $C_{0}(\Gamma)$ is Eberlein compact.

(b) $\Rightarrow$ (a). Talagrand (see [9] Theorem 4.3) has constructed a pointwise compact subset of $C_{1}(\Sigma)$ which is not Eberlein compact. Since for every closed subset $Y$ of $X, C_{1}(Y) \subseteq C_{1}(X)$, it follows that no closed subset of $X$ is homeomorphic to $\Sigma$. Because $X$ is analytic, it follows from theorem of Hurewicz (see [10], Corollary 1.4.5) that $X$ is $\sigma$-compact.

I don't know if the assumption that $X$ is analytic in condition (b) of Theorem 3.2 can be dropped. We note in this connection that the pointwise compact subsets of spaces of the form $C_{1}(X)$ where $X$ is a countably determined topological space are studied in detail in $[7,8]$.

Finally, we show that the spaces of the form $C_{1}(X)$ are hardly ever Grothendieck.

Proposition 3.4. For a separable metric space $X$ the following are equivalent:

(a) $C_{1}(X)$ is a Grothendieck space;

(b) $X$ is discrete $\left(\right.$ so $C_{1}(X)$ is identified with $\left.l^{\infty}(X)\right)$. 
Proof. (a) $\Rightarrow$ (b) We shall prove that every compact subset of $X$ is finite. So let $K$ be compact. Define $T: C_{1}(X) \rightarrow C_{0}(K) \subseteq C_{1}(X)$ with $T(f)=f \mid K$. Obviously, $T$ is bounded linear projection and $\|T\|=1$. Since $C_{1}(X)$ is Grothendieck and $C_{0}(K)$ is weakly compactly generated (see [3], Corollary 5, page 350) it follows that $T$ is a weakly compact operator. Therefore, if $B_{X}$ and $B_{K}$ denote the closed unit balls of $C_{1}(X)$ and $C_{0}(K)$, respectively, then $T\left(B_{X}\right)=B_{K}$ is weakly compact. It follows that $C_{0}(K)$ is reflexive, and so $K$ is finite.

(b) $\Rightarrow$ (a) By the remarks before Lemma 1.4, for every set $X$ the space $l^{\infty}(X)$ is a Grothendieck space.

REMARK. Since every injective Banach space is Grothendieck and $l^{\infty}(X)$ is injective, (a) and (b) of the above proposition are also equivalent to:

(c) $C_{1}(X)$ is injective.

\section{REFERENCES}

[1] J. P. R. Christensen, Necessary and sufficient conditions for the measurability of certain sets of closed subsets, Math. Ann., 200 (1973), 189-193.

[2] F. K. Dashiell and J. Lindenstrauss, Some examples concerning strictly convex norms on $C(K)$ spaces, Israel J. Math., $16(1973), 329-342$.

[3] J. Diestel, Geometry of Banach Spaces, Selected Topics, Lecture Notes in Mathematics 485, Springer-Verlag, Berlin, 1975.

[4] N. Dunford and J. T. Schwartz, Linear Operators I, General Theory, Pure and Appl. Math., Vol. 7, Interscience, New York, 1958.

[5] A. Grothendieck, Sur les applications linéaires faiblement compactes d'espaces du type $C(K)$, Canad. J. Math., 5 (1953), 129-173.

[6] K. Kuratowski, Topology, Vol. I, Academic Press, 1966.

[7] S. Mercourakis, Corson-compact spaces and the structure of weakly K-analytic Banach spaces, Doctoral dissertation, Athens University, 1983, (in Greek).

[8] On weakly countably determined Banach spaces, Trans. Amer. Math. Soc., to appear.

[9] M. Talagrand, Espaces de Banach faiblement K-analytiques, Annals of Math., 110 (1979), 407-438.

[10] F. Topsøe and J. Hoffman-Jørgensen, Analytic Spaces and Their Applications, In Analytic Sets, Academic Press, London, 1980.

[11] K. Yosida, Functional Analysis, Sixth edition, Grundlehren der. Math. Wiss. 123, Springer Verlag, Berlin, 1980.

Received April 25, 1985 and in revised form July 31, 1986.

ATHENS UNIVERSITY

PANEPISTEMIOPOLIS

15781 ATHENS, GREECE 


\section{PACIFIC JOURNAL OF MATHEMATICS EDITORS}

\author{
V. S. VARADARAJAN \\ (Managing Editor) \\ University of California \\ Los Angeles, CA 90024 \\ HERBERT Clemens \\ University of Utah \\ Salt Lake City, UT 84112 \\ R. FINN \\ Stanford University \\ Stanford, CA 94305
}

HERMANN FLASCHKA

University of Arizona

Tucson, AZ 85721

RAMESH A. GANGOLLI

University of Washington

Seattle, WA 98195

VAughan F. R. JONES

University of California

Berkeley, CA 94720

ROBION KIRBY

University of California

Berkeley, CA 94720
C. C. MoOrE

University of California

Berkeley, CA 94720

H. SAMELSON

Stanford University

Stanford, CA 94305

HAROLD STARK

University of California, San Diego

La Jolla, CA 92093

\section{ASSOCIATE EDITORS}
R. AREnS
E. F. BECKENBACH
B. H. NEUMANN
F. WOLF
K. YOSHIDA (1906-1982)

\section{SUPPORTING INSTITUTIONS}

UNIVERSITY OF ARIZONA

UNIVERSITY OF BRITISH COLUMBIA

CALIFORNIA INSTITUTE OF TECHNOLOGY

UNIVERSITY OF CALIFORNIA

MONTANA STATE UNIVERSITY

UNIVERSITY OF NEVADA, RENO

NEW MEXICO STATE UNIVERSITY

OREGON STATE UNIVERSITY
UNIVERSITY OF OREGON UNIVERSITY OF SOUTHERN CALIFORNIA STANFORD UNIVERSITY UNIVERSITY OF HAWAII UNIVERSITY OF TOKYO UNIVERSITY OF UTAH WASHINGTON STATE UNIVERSITY UNIVERSITY OF WASHINGTON 


\section{Pacific Journal of Mathematics}

\section{Vol. 128, No. $1 \quad$ March, 1987}

Anthony Peter Bahri and Peter Gilkey, The eta invariant, $\operatorname{Pin}^{c}$ bordism, and equivariant $\operatorname{Spin}^{c}$ bordism for cyclic 2-groups $\ldots \ldots \ldots \ldots \ldots \ldots 1$

Friedrich-Wilhelm Bauer, Extensions of generalized homology theories . . . 25

Marilyn Breen, A characterization theorem for compact unions of two

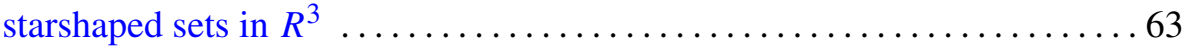

Boniface Ihemotuonye Eke, Special generating sets of purely inseparable extension fields of unbounded exponent $\ldots \ldots \ldots \ldots \ldots \ldots \ldots \ldots \ldots \ldots$

Robert William Gilmer, Jr. and William James Heinzer, Jónsson $\omega_{0}$-generated algebraic field extensions $\ldots \ldots \ldots \ldots \ldots \ldots \ldots \ldots \ldots . . .61$

Guido Lupacciolu, Holomorphic continuation in several complex variables

Douglas C. McMahon, Jaap C. S. P. van der Woude and Ta-Sun Wu,

Connectedness related to almost periodicity of compositions of flow

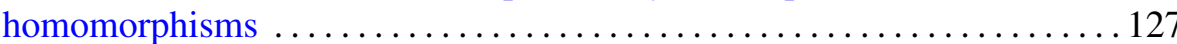

Sophocles Mercourakis, Some characterizations of analytic metric spaces

Peter Frederick Stiller, The Picard numbers of elliptic surfaces with many

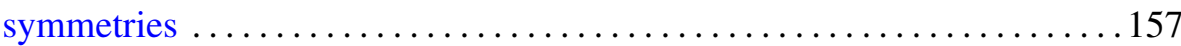

David J. Winter, Reducible complements of Lie algebra radicals ........ 191 Rade Živaljević, On a cohomology theory based on hyperfinite sums of microsimplexes 